\title{
Ventilación Mecánica en Insuficiencia Respiratoria de Origen Pulmonar
}

\author{
Dr. Mario Cerda S. ${ }^{1}$; Dr. Rolando Saavedra $O^{1}{ }^{1} ; 3$; Dr. Manuel Aspillaga E. ${ }^{2}$; Dr. Tomás Mesa L. ${ }^{2}$; Fnf. Sara Peña \\ $\mathrm{R}^{1}$; Kin, Elizabeth Arenas $\mathrm{G},{ }^{1}$
}

\section{MECHANICAL VENTILATION IN RESPIRATORY FAILURE OF PULMONAIRE ORIGIN}

A retrospective analysis of 26 children admitted during 1980 and 1981 with acute respiratory failure (A.R.).) caused,by Bronchiolitis and Brochopneumonia and manged with Mechanical Ventilation (V.V.) is presented. Within this group the majority of the children were younger than 1 years of age $(22 / 26)$, well nourished (19/26) and 11 had antecedents of low birth weight or premature delivery. The most frecuent indication for $M$. V. was brochopneumonia (21/26). The mean hospitalization period was 35,6 days even if in $88 \%$ of the patients (23/26) the duration of M.V. was less than 7 days. In this series the mortality rate was $23 \%$. At the discharge $65 \%$ \% (17/26) were asymptomatic or had minor respiratory symptoms and $11,5 \%$ \% (3/26) were considered with chronic pulmonary damage.

Las enfermedades respiratorias siguen tenien. do una alta incidencia en nuestro país ${ }^{1}$ y afectan de preferencia y con mayor gravedad las primeras edades de la infancia. Son el principal componente de la mortalidad infantil, alcanzando el 5,4 por 1.000 de los nacidos vivos ${ }^{1}$. En Chile fallecen anualmente 1.300 menores de 1 año por bronconeumonia; si ellos hubiesen sido favorecidos con técnicas de tratamiento más avanzadas y especializadas, dichas cifras disminuirian de manera importante, como, en otro nivel, ha ocurrido con la mortalidad por patología respiratoria del recién nacido después de la organización de Unidades de Cuidado Intensivo Neonatales.

Lactantes y preescolares en cambio no han sido analizados con detención, ya que no existen programas especiales de implementación humana e instrumental para su tratamiento en Unidades de Cuidados Intensivos Pediátricos (UCI.P.).

En nuestro medio no hay publicaciones de las experiencias y resultados de los centros que actualmente estân en condiciones de manejar la Ventilación Mecánica (V.M.) en Pediatría. Esta es una técnica muy útil en el tratamiento, de pa-

1 Hospital Sótero del Río. Servicio de Pediatria (U.C.I.P.).

2 Médico becario, Departamento de Pediatría, Lniversidad Católica de Chile.

3 Universidad Católica de Chile. cientes con cuadros respiratorios graves, que si se lleva a cabo en el momento oportuno y en forma adecuada puede, sin lugar a dudas salvar a muchos nin̄os afectados de Insuficiencia respiratoria aguda (I.R.A).

\section{MATERIAL Y METODO}

Se revisó en forma retrospectiva 26 fichas de pacientes que fueron tratados con V.M. a raiz de una I.R.A., de origen exclusivamente pulmonar (Bronconeumonias y Bronquitis Obstructiva), durante el lapso de tiempo comprendido entre Enero 1980 y diciembre 1981.

Se excluyó aquellos casos que, además de I.R.A., presentaban otro hecho agregado (sepsis, shock, etc...), asi como tambiến aquellos cuya patología pulmonar apareció en el curso de otra enfermedad (meningitis, cardiopatias, etc...), o los derivados del uso del ventilador. Tampoco se consideró a pacientes con Laringitis Obstnuctiva tratados con adrenalina racémica.

Los siguientes parámetros fueron analizados: edad, sexo, estado nutritivo según Sempé ${ }^{2}$, antecedentes mórbidos perinatales y patolog ía respiratoria previa, diagnóstico de egreso, fundamento $y$ duración de la V.M., días de hospitalización general y en LCl.P., complicaciones, estado clínico al alta y mortalidad.

En la UCI.P. se pueden medir los gases sangui- 
neos las 24 horas del día para el.manejo de estos pacientes.

Cuando se decide conectar a un paciente se le ventila previamente por 5 minutos con oxígeno $100 \%$ por intermedio de Ambú, se administra Valium (R) $(0,5 \mathrm{mg} . \mathrm{Kg} / \mathrm{iv}$ ), Atropina $(0,01 \mathrm{mg}$ ! $\mathrm{Kg} / \mathrm{iv}$ ) y Succinil colina $0,8 \mathrm{mg} / \mathrm{Kg} / \mathrm{iv}$.

Se prefiere la intubación nasotraqueal con cánulas de clonuro de polivinilo, ya que su manejo resulta fácil y rápido, permite una excelente fijación y disminuye algunas complicaciones ${ }^{3-4}$.

Para efectuar tratamiento de V.M. se contó con un Ventilador Bird Mark $8^{5}$ y un Bennet PR - 2, que fueron empleados indistintamente, de acuerdo a su disponibilidad, con circuito estéril (en oxido de etileno), que fue cambiado cada 8 horas. Para el nebulizador se usó agua bidestilada estéril que se cambió cada 12 horas. Se tomó cultivo de secreción traqueal cada 48 horas. Se aspiró al paciente cada 1 o 2 horas con intilación previa de 1 a $2 \mathrm{ce}$., de solución de $\mathrm{NaCl}$ al $9 \%$ estéril mediante sonda y bandeja estéril ${ }^{6}$.

La kinesioterapia respiratoria (KTR) fue practicada por Kinesiólogo 2 veces al día los días hábiles, y por médico residente o enfermera los dias festivos y en horas nocturnas. Se cambió la posición de drenaje postural cada 2 horas ${ }^{7}$. El antibiótico para tratar las Bronconeumonias fue elegido según resultados del cultivo de secreción traqueal y antibiograma, aunque antes de conocer este último, se indicó uno de amplio espectro.

Diagnosticamos atelectasia pulmonar ante un aumento brusco de] corto circuito intrapulmonar, clínica de condensación sin crépitos y radiografia pulmonar compatible. En el caso de intubación monobronquial la sospechamos clínicamente por hipoventilación y asimetria en la excursión torácica, y se comprobó radiológicamente.

El criterio seguido para retirar el ventilador fue un cortocircuito intrapumonar menor del $20 \%$ y ausencia de retención de $\mathrm{CO}_{2}$, buen esfuerzo respiratorio, ventilación mandatoria in. termitente (V.M.J.) con frecuencias bajas (menos de 8 por minuto $)^{a-9-10}$.

Para el cálculo rápido y aproximado del corto circuito intra pulmonar se usó la curva de Pontoppidan (Fig. 1).

Todas estas técnicas y normas han sido.fijadas e implementadas progresivamente. Concentramos el análisis sólo en los últimos dos años, en que el tratamiento fué uniforme.

\section{RESULTADOS}

Durante el periodo 1980 - 1981, se hospitalizaron 512 pacientes en UCI.P., y de estos, 98 $(19.10 \%)$ requirieron V.M.
Sólo a 26 pacientes se les indicó V.M. debido a una patología pulmonar exclusiva, concentrándose en ellos la presente comunicación.

Se ubicaron 25 fichas $y$ por este motivo la ficha No 26 sólo se considera dentro del análisis de sexo, mortalidad y duración de V.M. para no alterar las cifras estad ísticas.

Los fundamentos para indicar V.M. en los pacientes estudiados fueron insuficiencia respirato-

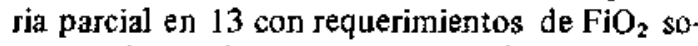
bre $60 \%$. En la mitad de estos enfermos se consigna en la ficha un agotamiento respiratorio clinico.

Analizando algunos parámetros encontramos que la gran mayoría de los pacientes corresponde a lactantes (19 pacientes) (Tabla 1), su sexo fue de masculino en $19(73 \%)$ y femenino en 7 $(27 \%$ )., la gran mayoría eran niños eutropi$\cos ^{12}$ o desnutridos leves (11 pacientes) y sólo había 2 desnutridos de 3 er. grado.

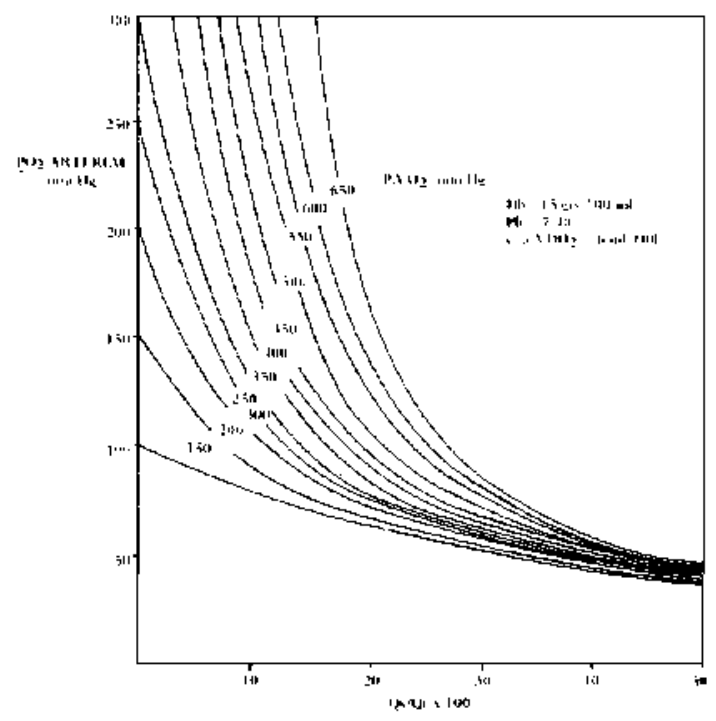

Fig. 1. Relación entre $\mathrm{PO}_{2}, \mathrm{PAO}_{2}$ y corto circuito intrapulmonar (Según Pontoppidan), Conociendo $\mathrm{PO}_{2}$ y $\mathrm{PAO}_{2}\left(\mathrm{PAO}_{2}=\mathrm{FiO}_{2}\right.$ (Patmosf $\left.\left.-\mathrm{PH}_{2} \mathrm{O}\right)-\mathrm{PCO}_{2}\right)$.

Se puede deducir en forma aproximada el Porcentaje de Corto circuito intrapulmonar. (Qs/Qt x 100).

Tabla 1.

Edad de los Pacientes

Edad

$\begin{array}{lrr}\text { Edad } & \begin{array}{c}\text { Número } \\ \text { pacientes }\end{array} & \text { \%/o } \\ \text { Recién nacidos } & 3 & 12 \\ \text { Lactantes menores } & 19 & 76 \\ \text { Lactantes mayores } & 2 & 8 \\ \text { Preescolares } & 1 & 4 \\ \text { Total } & 25 & 100\end{array}$


Once pacientes tenían antecedentes de prematurez, bajo peso al nacer o ambos $(<2.500 \mathrm{~g}$.$) ;$ Cuatro niños habian sido hospitalizados previamente por cuadros respiratorios y sólo una habia necesitado de V.M. en el periodo de recién nacidos.

El tiempo transcurrido desde el inicio de la enfermedad respiratoria hasta el uso de V.M. fue en 20 casos de 7 dias o menos, en 2 entre 8 y 14 dias y en 3 de más de 15 días. El diagnóstico de egreso fue sindrome bronquial obstructivo en $3 \mathrm{pa}$ cientes, cuerpo extraño laringeo y bronconeumonia en uno, bronconeumonia en $21(84 \%)$.

En su mayoría los pacientes fueron tratados por menos de 7 días (Tabla 2) con ventilación mecánica.

Tabla 2.

Duración de la Ventilación Mecánica

$\begin{array}{lcr}\text { Número días } & \begin{array}{c}\text { Número } \\ \text { pacientes }\end{array} & \% \% \\ 2 \text { días o menos } & 13 & \mathbf{4 8} \\ 3-7 \text { días } & 10 & \mathbf{4 0} \\ 8-20 \text { dias } & 0 & 0 \\ 21 \text { días omás } & 3 & 12\end{array}$

El promedio de días de hospitalización fue de 35,6 días y la permanencia de UCI.P. de 15,26 dias por paciente. Cinco niños estuvieron más de 60 y uno de ellos 132 días en el hospital.

Las complicaciones más frecuentes se detallan en la Tabla 3.

Tabla 3.

Complicaciones de Ventilación Mecánica

- Arelectasia

- Intubación moлobronqujal

- Incoordinación musculas

- Distensión abdominal

- Extubación

Falla del ventilador

- Enfiscma subcutáneo

- Neumo mediastino

- Tapón de cánula

- Laringitis postextubación

En el momento del egreso, de 26 pacientes, 6 fallecieron $(23 \% / 0) ; 12(46,19 \%)$ estaban asintomáticos desde el punto de vista respiratorio; 5 $(19,2 \%)$ presentaban signos respiratorios leves y $3(11,5 \%)$ fueron considerados con signos importantes. En la figura 2 se describe la mortalidad de la serie en comparación con la encontrada en el total de pacientes ventilados durante el mismo periodo en la UCI.P.
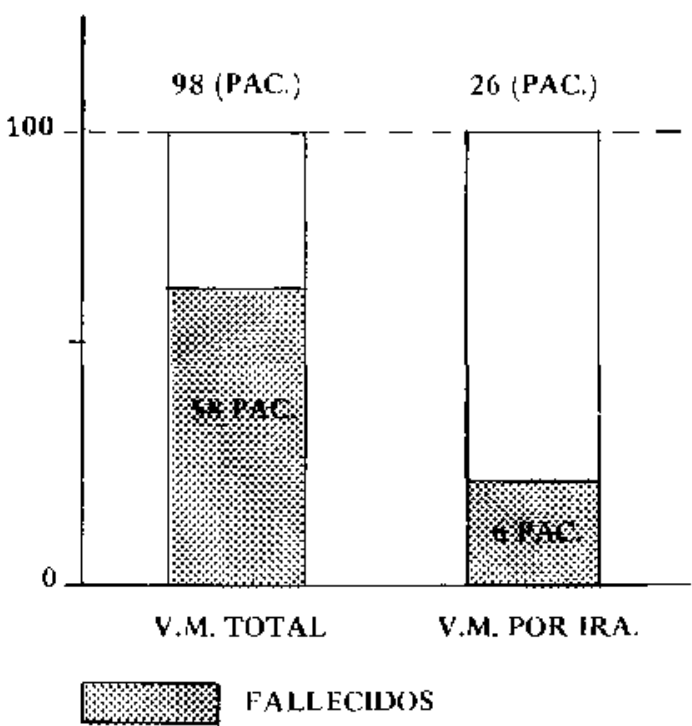

(PAC.) NO DE PACLENTES

Fig. 2: Comparación de Mortalidad de Ventilación Mecánica según patologías: general y con patología pulmonar exclsuvia.

\section{COMENTARIO}

La proporción de pacientes hospitalizados en UCI.P. fue de $11,44 \%$ o del total de los ingresos al Servicio de Pediatría del Hospital Sótero del Rio, durante el periodo 1980.1981 , to que demuestra la necesidad de contar con este tipo de Unidades ${ }^{11}$.

Llamó la atención la alta incidencia de l.R.A. en lactantes menores de un año, lo que atribuimos a sus características anatomofísiológicas que los hacen más susceptibles de presentar estos cuadros graves $y$ de caer en I.R.A. ${ }^{12-13-14-15-16-17}$ En concordancia con otras publicaciones, el sexo masculino tiene mayor morbimortalidad respiratoria en estas edades ${ }^{18}$. En cuanto el estado nutritivo, el hecho de encontrar un $8 \%$ o de desnutrición Gr. III en estos pacientes indicaria una pre. disposión a presentar I.R.A. en ellos, ya que en la población menor de 6 años de nuestra área, representan sólo el $0,1 \%$ del total ${ }^{11}$.

El antecedente importante de la alta incidencia de recién nacidos prematuros o de bajo peso apoya la jdea que ellos deben tener un seguimiento cuidadoso y ser considerados de alto rjesgo para patologia respiratoria.

Llamó la atención que la mayoría de los pacientes tenia una enfermedad respiratoria de evolución menor de 7 días antes de la jndicación de ventilación mecainica, periodo suficiente para llevarlos a una I.R.A., lo que indica que deben ser vigilados y tratados en una unidad especial de cuidados respiratorios. 
En nuestra unidad, la mayoría de los ninos que necesitaron V.M., por causa respiratoria tenían broconeumonia, a semejanza de lo que ocurre en otros países de mayor desarcollo ${ }^{19}$.

Hicimos una distribución arbitraria del tiem. po requerido de V.M., comprobando que la gran mayoría de los pacientes necesitó menos de 7 dias, to que se explica por las caracteristicas anatomopatológicas y agudeza de una bronconeumo$\mathrm{nia}^{20}$.

El promedio de días de hospitalización para enfermos con esta patología es mayor que el promedio general, que en nuestro Hospital es de 11,5 días. Esto se explica por la complejidad del tratamiento, gravedad del cuadro y sus complicaciones. Estas últimas son las clásicas que se describen. La gran mayoria fueron debidas al manejo de cánula. Hemos visto pocos barotraumas en comparación con la alta incidencia en la literatura $^{21-22}$, probablemente porque somos menos agresivos en el tratamiento y usamos presiones más bien bajas.

Las condiciones al alta fueron en general aceptables en relación a los cuadros que tratamos, lo que hay que evaluar con estudios funcionales a largo plazo, actualmente en marcha.

La mortalidad de nuestro grupo, considerando que estos pacientes, tenian una indicación ineludible de V.M., sin la cual la gran mayorja habria fallecido, fue menor de un $25 \%$, to que ya es un logro importante. Estamos convencidos que ella se puede reducir aún más perfeccionando las técnicas, entrenando mejor al personal y usando los medios adecuados. Si pensamos que un alto porcentaje de estos pacientes son nirios nomnales y eutróficos, todo esfuerzo en este campo debe apresurarse.

\section{RESUMEN}

Se efectúa un análisis retrospectivo de fichas de niffos con Insuficiencia Respiratoria Aguda (I.R.A.) de origen exclusivamente pulmonar que necesitan Ventilación Mecánica (V.M.) durante el periodo 1980 y 1981 . Se encuentra un predominio de lactantes menores con antecedentes de prematurez y eutróficos en el momento de enfermar. La causa más frecuente de I.R.A. fue la bronconeumonia, en general necesitaron V.M., por menos de 7 dias $y$ en promedio estuvieron hospitalizados por 36,6 días. La mortalidad de nuestra serie fue de $230 \%$ y de los que no fatlecen, la gran mayoría se encontraban asintomáticos en el momento del alta.

\section{REFERENCIAS}

1 COMPAN: Acciones en Desarrollo. Ministerio de Salud, 1978.

2 Sempé, M.; Roy, M.P., Pedron, G.: Pediatrie Sociale, 1) lammarion Medicine-Sciences, Paris, 1972.

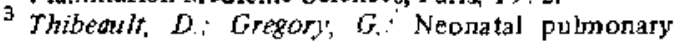
Care. Ed. Eddison-Wesley Publishing Company, California, 1979.

4 Gross, C.W.: Medical management nasotracheal inturbation and tracheostomy in the treatment of upper airuay. Obstruction in children. Otolaryngol. Clin. North Amer. 10: No 1 ( (t'eb), 1977.

5 Mc. Pherson, S.: Respiratory therapy equipment, 2nd. Ed. The C.V. Mosby Company, St. Louis, 1981, Chapt. 9.

6 Morrison, M.1.: Respiratory Intensive Care Nursing, Ed. Little Brown and Company, Boston, 1979.

7 Zoilar, L.M.: Krausse, H.E., Mufson, M.A.: Microbiologic studies on young infants with lower respiratory disease. Am. J. Dis. Child. 126:56, 1973.

${ }^{8}$ Gregory, G.A.; Respiratory Failure in the Chitd. Ed. Churchill Livingstone, New York, 1981.

9 Newth, $C_{\text {: }}$ Recognition and management of respiratory Failure. Ped. Clin. N.A. 26: 617, 1979. 1979 .

${ }^{10}$ Morrison, M.L.: Respiratory Intensive Care Nursing, Ed. Little Brown and Company, Boston, 1979.

11 Servicios Estadisticas. Area Metropolitana Sur Oriente de Salud.

12 Holsclow, D.S.: Early Recognition of acute Respiratory Vailure in Children. Pediatr. Ann. 6:57, 1977.

13 Kendig. E.L.: Alteraciones de las vías respiratorias en los niños. Vol. l. Salvat Ediciones, Barcelona, 1977.

14 Green, G.M.: Defense Mechanism of the Respiratory Membrane, Amer. Rey, Resp. Dis. 115:479, 1977.

15 Newhouse, M.: Lung defense mechanism. N. Engl. J. Med. 295:1045, 1976, 295:990, 1976.

16 Johnson, K.J.: Immunopattrolugy of the lung. Amer. J. Pahtol 95:795, 1979.

17 Schtz. M.: Inmunologic lung Diseases. N. Engl. J. Med. 300:1310, 1979.

18 Schatfer, A.; Avery, M.E.: Discases of the newborn. 4 th. Edit. W.B. Sauders Company, Philadelphia, 1977, Chapt 84

19 Gregory, G.A.: Respiratory liaiture in the Child. Chapt. 3, Ed. Churchill Livingstone, New York, 1981.

20 Robins, $S$.: Texbook of Pathology. Chapt. 19, W.S. Sau nders Co. Philadelphia, 1964.

21 Thibeoul, D.W.: Zachman, R.S.; Zaul, V.R.: Pulmonary intersticial emphysema, pneumomediastinum and pneumpthorex. Am. J. Dis. 126:611, 1973.

22 Thibeoult, D.W.: Gregory, G.A.: Neonatal Pulmonary Care. Ed. Addison-Wesley, Chapt. 20, Massachusetts, 1979. 